\title{
Transforming a large introductory lab course: impacts on views about experimental physics
}

\author{
Benjamin Pollard ${ }^{1,2}$ and H. J. Lewandowski ${ }^{1,2}$ \\ ${ }^{1}$ Department of Physics, University of Colorado Boulder, Boulder, CO 80309, USA \\ ${ }^{2}$ JILA, National Institute of Standards and Technology, Boulder, CO 80309, USA
}

\begin{abstract}
Laboratory courses are key components of most undergraduate physics programs. Lab courses often aim to achieve the following learning outcomes: developing students' experimental skills, engaging students in authentic scientific practices, reinforcing concepts, and inspiring students' interest in physics. Some of these outcomes can be measured by the Colorado Learning Attitudes about Science Survey for Experimental Physics (E-CLASS), a research-based assessment on students' views about experimental physics. We used E-CLASS at the University of Colorado Boulder to measure learning outcomes during a course transformation in which views about experimental physics were reflected in the learning goals. We collected over 600 student responses per semester from the large introductory laboratory course, both before and after implementing the course transformation. While we observed no statistically significant difference in overall post-instruction E-CLASS scores before and after the transformation, in the transformed course, student responses to three E-CLASS items that related to the goals of the transformation were more favorable than in the original course.
\end{abstract}

\section{INTRODUCTION}

Science and technology are rooted in data and observation, and the field of physics is no exception. Regardless of their subfield or specialization, a primary way experienced physicists evaluate their theories and ideas is by comparing them to empirical evidence [1]. Furthermore, such evidence is often the inspiration for new theories and ideas themselves [1]. Therefore, physics students must gain an understanding of experimental techniques, equipment, processes, and habits of mind in order to better align their practices with those of expert physicists. That learning occurs primarily in lab courses [2]. As such, there is growing national interest in studying, investing in, and improving lab courses [3, 4].

In particular, lab courses are one of the only opportunities in undergraduate physics curricula for students to develop their views about experimental physics [5]. We define "views about experimental physics" to include attitudes, beliefs, and expectations about the nature and importance of experimental physics, as well as the strategies, emotions, and habits of mind that are involved when doing physics experiments. Instructors of lab courses often mention the importance of students developing their views about experimental physics [6]. Consequently, learning goals of lab courses often include outcomes related to students' views about experimentation [7].

With the above ideas in mind, we have led a research-based transformation of the introductory lab course at the University of Colorado Boulder (CU). We identified a set of learning goals with input from STEM faculty at CU as an early step of the transformation process. In addition to goals regarding measurement uncertainty $[8,9]$ and scientific communication, more than one learning goal focused explicitly on views about experimental physics. Prior to the course transformation, we collected data from the original course for several semesters along dimensions aligned with these learning goals, in order to establish a baseline of comparison for the transformation. The same data were collected in Spring 2018 during the first semester of the transformed course.

Here, we focus on the following research question: To what extent did our course transformation affect students' views about experimental physics? To answer that question, we use data from the Colorado Learning Attitudes about Science Survey for Experimental Physics (E-CLASS), a research-based assessment tool for undergraduate lab courses [5, 10-12]. We compare E-CLASS results from the original and transformed courses.

\section{CONTEXT AND METHODS}

In this section, we provide details about (A) the course context both before and after transformation, (B) the E-CLASS assessment tool, and (C) our student population and methods.

\section{A. Course context}

The course studied here is the first physics lab course that students take at CU, typically in their second semester of study. It is a large-enrollment stand-alone lab course worth one credit (typical courses at CU are worth three credits), usually taken concurrently with a separate physics lecture course. The lab course, both before and after our transformation, consists of a series of weekly two-hour lab activities involving mechanics, electricity and magnetism, optics, and other physics concepts, all at an introductory level. There is no midterm or final exam for the course; grades are based primarily on work produced from the weekly activities and participation in occasional supplemental lecture sessions.

Prior to the course transformation, students rotated through a series of lab activities, and different students worked on different activities at the same time. Students performed measurements together, but were required to work independently when analyzing data and writing reports in order to reduce cheating. Many of those activities involved measuring a known physical parameter already familiar to students, for example, using a pendulum to measure the acceleration due to gravity at the earth's surface.

Our course transformation process was initiated in Spring 2016. Faculty members and staff selected from physics, engineering, and applied science departments were interviewed to develop consensus learning goals for the course. Five 
learning goals emerged, including "Students' epistemology of experimental physics should align with the expert view," and "Students should demonstrate an expert-like understanding of measurement uncertainty when evaluating measurements." Each learning goal was associated with an assessment tool, research-based if possible, for measuring corresponding learning outcomes. Further details of the course transformation process can be found in Ref. [13].

Each lab activity and lecture was completely redesigned as part of the course transformation. The lectures were also updated to match the new lab activities and overall course content. Nonetheless, many aspects of the transformed course remained the same or similar to the pre-transformed version, making the resulting course still "traditional" in the following ways. Students still completed a pre-lab assignment before class, attended weekly lab sessions in which they worked through an activity guided by a manual, and attended occasional lectures.

In the transformed lab sessions, all students did the same activity in a given week, mostly working in pairs or groups of three. The activities were designed to encourage interaction between students, often prompting students to check with their partner or another group before continuing, and sometimes requiring students to combine their data with the rest of their lab section. In most of the activities, students did not know the numerical result of their measurement before it was made. Instead, students were guided to make preliminary measurements of relevant parameters, and then to use those measurements to make predictions or measure unknown quantities. For example, in one activity, students calibrated the change in capacitance of a parallel plate capacitor as known masses were placed on top of it, and then used those data to measure the weight of an unknown mass. The activities often involved the explicit use of measurement uncertainty concepts, both to make predictions and compare data. Instead of writing lab reports, students created electronic lab notebooks during lab, and uploaded those notebooks before they left their session.

\section{B. E-CLASS assessment tool}

We use E-CLASS to measure student learning related to our transformation learning goals around attitudes and beliefs about experimental physics [14]. E-CLASS was developed in our research group at $\mathrm{CU}$ "with the goal of providing a quantitative measure of the effectiveness of [course] transformations [5]." The assessment consists of 30 statements about experimental physics, and asks students to rate their level of agreement with each statement on a 5-point Likert scale. Each statement has a defined expert-like response, confirmed and validated by the consensus of over 20 practicing experimental physicists and lab instructors [12]. While the assessment asks students to respond both from their own perspective and from that of a hypothetical experimental physicist, we only consider the responses from the students' perspectives here.

E-CLASS measures students' beliefs, attitudes, and expec- tations about the nature of experimental physics. This scope includes the importance of experimentation in the field of physics as a whole, the habits of mind employed while measuring and experimenting, students' emotional relationship with experimental physics, and the roles of common practices such as identifying research questions, making predictions, understanding equipment, fixing problems, interpreting raw data, and communicating results [10].

\section{Student population and study methods}

The introductory physics lab course is one of the largest physics courses offered at CU. Its enrollment has been steadily increasing over the past several years, with over 700 students in the course in Spring 2018. Table I shows demographic information of the students enrolled in Spring 2018.

TABLE I. Self-reported gender, race, ethnicity, and major of students enrolled in the course in Spring 2018.

\begin{tabular}{r|r}
\hline \hline Female & $25.6 \%$ \\
Male & $72.7 \%$ \\
Gender non-conforming & $1.7 \%$ \\
\hline American Indian or Alaska Native & $0.7 \%$ \\
Asian American & $15.3 \%$ \\
Black or African American & $2.3 \%$ \\
Hispanic/Latino & $9.8 \%$ \\
Native Hawaiian or other Pacific Islander & $0.4 \%$ \\
White & $67.1 \%$ \\
Other race/ethnicity & $4.3 \%$ \\
\hline Physics & $17.9 \%$ \\
Other Engineering & $43.0 \%$ \\
Other STEM & $35.1 \%$ \\
Other disciplines & $4.0 \%$ \\
\hline \hline
\end{tabular}

E-CLASS was administered online at the beginning and end of the semester, referred to respectively as pretest and post-test. Data collection was performed for several years leading up to the first implementation of the transformed course. Prior to the transformation, time was provided during regularly scheduled lab sessions for students to complete the assessment. E-CLASS was also administered at the beginning and end of the transformed course in Spring 2018. Due to time constraints, students in the transformed course completed the assessment outside of class using a link provided via email. However, previous studies have shown no statistical difference in students' matched responses between in-class and out-of-class administration of E-CLASS [15]. In all semesters, students received course credit for completing both the pretest and the post-test, amounting to about $1 \%$ of their final grade in total.

In order to account for variations between Fall and Spring semesters, in this study, we use data from only the Spring 2017 semester (before the transformation) to compare to Spring 2018 (after the transformation). In both of those semesters, the course was taught by the second author.

Data from E-CLASS was scored using established methods from previous studies [10]. The 5-point Likert scale re- 
sponses to each E-CLASS item were collapsed into a 3-point scale, in which "(dis)agree" and "strongly (dis)agree" were combined into a single category. A response aligned with expert-like views was assigned a score of +1 and referred to as favorable, whereas a response opposite of expert consensus was assigned a score of -1 and referred to as unfavorable. Neutral responses were assigned a score of 0 .

Here, we analyze responses to individual E-CLASS items, and also compute an overall score for each student by summing the student's scores on all 30 E-CLASS items. We compare the distributions of scores, both overall and itemby-item, using the nonparametric Mann-Whitney U test at the 5\% significance level to determine statistical significance. To account for confirmation bias arising from performing multiple item-wise comparisons, we apply the Bonferroni correction, treating each item as an individual hypothesis. We use Cohen's $d$ as a measure of effect size for statistically significant differences. See Ref. [16] for a complete description.

Before analyzing any E-CLASS data from this course, both authors read through the list of 30 E-CLASS items and identified the ones that were related to our course transformation goals. This subset of E-CLASS items was further discussed with the other instructor of the transformed course, resulting in a final set of nine items. Criteria for whether an item was related to the course transformation centered around whether there were specific lab activities, or specific design choices implemented throughout the course, that were intended to address the view probed by that item. We consider only these nine items in our item-by-item analysis below.

\section{RESULTS AND DISCUSSION}

We begin by comparing the distributions of overall scores before and after the course transformation, comparing pretest to pretest and post-test to post-test. The two distributions of overall pretest scores are statistically equivalent. This result confirms that the student populations were similar in their views about experimentation at the start of the course.

Since the two pretests showed similar results, we turn to the post-tests to better understand how students' views changed by the end of the course. We find that in overall score, the two post-test distributions are also statistically equivalent, suggesting that the course transformation did not have a marked effect on students' views about experimental physics when distilled into a single number. However, the scope that ECLASS probes is far broader than our relatively narrow transformation learning goals. Thus, we do not expect our transformation to have a large effect on overall E-CLASS scores.

To get a more fine-grained perspective on the effects of the course transformation, we analyze E-CLASS post-test scores item-by-item. The nine items related to our course transformation goals are shown in Table II, and plotted in Fig. 1. Scores are plotted for both the Spring 2017 and Spring 2018 semesters. Three of these items, those marked with black circles in the figure and bold in the table, showed statistically significant differences in post-test distributions before and after the transformation. The $p$-values used to determine sig-

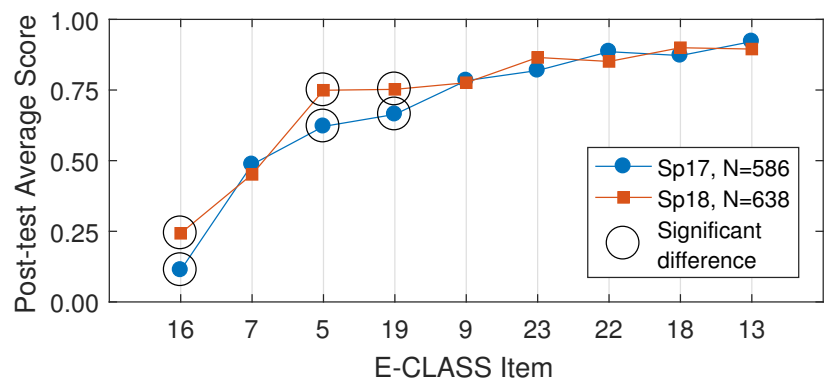

FIG. 1. Comparison of post-test scores on nine E-CLASS items related to transformation goals, averaged across all students in the course. Data from Spring 2017, before the transformation, is compared to data from Spring 2018, the transformed course. Items are ordered by the mean of the scores from the two semesters. Black circles mark the items that show statistically significant differences between semesters.

nificance are shown for all items in the table. The items with significantly different post-test distributions between the two semesters are highlighted in bold, and include corresponding $d$-values to measure effect size. These effect sizes have practical as well as statistical significance in the context of typical effect sizes from previous E-CLASS studies [10].

Among all nine items, we see no significant negative changes in average E-CLASS scores after the transformation, which suggests that the transformed course is not significantly worse than the original at promoting favorable views about experimentation along these nine dimensions. Furthermore, five of the six items that remained unchanged after the transformation all have average scores above 0.75 , indicating that these items had little room to improve due to the course transformation. The three items that did show significant change shifted towards more favorable responses after the transformation. We discuss each in detail below.

Item 16 concerns confirming previously known results. Note that the consensus expert response to this item is to disagree, as experiments usually serve a larger purpose than confirming previous results. As a design goal aimed at achieving our learning goals, activities with the purpose of confirming previously known results were deliberately avoided in the transformed course. The favorable shift in Item 16 after the transformation is likely related to this design goal. Previous research has shown that students can interpret the phrase "previously known results" as either refering to their personal scientific knowledge, or to well-established results from the scientific community [11]. In either case, a shift towards more favorable responses on this item represents a desirable outcome of the course transformation.

Item 5 concerns the role of measurement uncertainty in interpreting results. A more expert-like understanding of measurement uncertainty was a learning goal of the transformed course. The statistically significant shift in Item 5 suggests that, distinct from the concepts themselves, students' views about measurement uncertainty were affected favorably by the transformation. Previous work has shown that students in the original course already show pre-post shifts towards such 
TABLE II. E-CLASS items identified as related to our course transformation goals. Items in bold show statistically significant changes before and after the transformation. Order of items is the same as in Fig. 1.

\begin{tabular}{|c|c|c|c|}
\hline$\overline{\text { Number }}$ & Statement & $p$ & $\bar{d}$ \\
\hline 16 & The primary purpose of doing physics experiments is to confirm previously known results. & 0.005 & 0.164 \\
\hline 7 & I don't enjoy doing physics experiments. & 0.339 & \\
\hline 5 & Calculating uncertainties helps me understand my results better. & 0.001 & 0.207 \\
\hline 19 & Working in groups is an important part of doing physics experiments. & 0.005 & 0.156 \\
\hline 9 & When I approach a new piece of lab equipment, I feel confident I can learn how to use it well enough for my purposes. & 0.759 & \\
\hline 23 & When I am doing an experiment, I try to make predictions to see if my results are reasonable. & 0.085 & \\
\hline 22 & $\begin{array}{l}\text { If I am communicating my results from an experiment, my main goal is to make conclusions based on my data using } \\
\text { scientific reasoning. }\end{array}$ & 0.066 & \\
\hline 18 & Communicating scientific results to peers is a valuable part of doing physics experiments. & 0.124 & \\
\hline 13 & If I try hard enough, I can succeed at doing physics experiments. & 0.104 & \\
\hline
\end{tabular}

conceptial understanding [8,9]. Additional work is needed to see whether conceptual understanding also improves in the transformed course and, if so, how students' views about and their understanding of measurement uncertainty are coupled.

Lastly, Item 19 concerns the importance of working in a group when doing physics experiments. Group work was required in the transformed course, in stark contrast to the focus of the original course on individualized work. Furthermore, the activities in the transformed course often prompted students to compare and combine data with the entire class, promoting interactions between students specifically about their measurements. These aspects plausibly contributed to the significant favorable shift in this E-CLASS item.

\section{CONCLUSIONS AND OUTLOOK}

Compared to the original course, our transformed course did not yield a statistically different distribution of overall E-CLASS post-test scores. However, on particular items related to the goals of the transformation, three showed statistically significant post-test differences after the transformation. These items related to views about confirming known results, about measurement uncertainty, and about working in groups. The three shifts were towards more expert-like responses.

Future work will involve a deeper analysis of E-CLASS data from both the original and the transformed course, and will compare E-CLASS findings to results from a variety of other assessment tools and research methods. The transformed course will be further improved based on those findings, and provide opportunities for further study. We expect that these data sets, when analyzed together, will provide a more complete picture of the effects of our transformation on student learning, and provide a blueprint for transforming and evaluating lab courses more generally. As researchers further understand student experiences in physics lab courses, they will be able to build on existing opportunities to better support the unique learning that is possible in physics labs.

\section{ACKNOWLEDGMENTS}

We acknowledge D. Bolton, R. Hobbs, C.West, S. Woody, M. Schefferstein, A. Ellzey, M. Dubson, and J. Bossert for their work in developing the transformed course. We also acknowledge D. Dounas-Frazer and L. Ríos for their input during the course transformation, B. Wilcox for her help with E-CLASS data analysis, and the many student testers for their contributions to improving the labs. This work is supported by the NSF under grant PHYS-1734006, the office of the Associate Dean for Education of the College of Engineering and Applied Science and the College of Arts and Sciences at the University of Colorado Boulder.
[1] I. T. Koponen, Science \& Education 16, 751 (2007).

[2] M. D. Caballero, D. R. Dounas-Frazer, H. J. Lewandowski, and M. R. Stetzer, APS News Back Page 27 (2018).

[3] National Research Council, (2012), 10.17226/13362.

[4] AAPT Committee on Laboratories, AAPT (2014).

[5] B. R. Wilcox and H. J. Lewandowski, Am. J. Phys. 86, 212 (2018).

[6] D. R. Dounas-Frazer and H. J. Lewandowski, Phys. Rev. PER 13, 010102 (2017).

[7] D. R. Dounas-Frazer and H. J. Lewandowski, PERC Proceedings , 100 (2016).

[8] B. Pollard, R. Hobbs, J. T. Stanley, D. R. Dounas-Frazer, and H. J. Lewandowski, PERC Proceedings , 3 (2017).

[9] H. J. Lewandowski, R. Hobbs, J. T. Stanley, D. R. Dounas-
Frazer, and B. Pollard, PERC Proceedings , 244 (2017).

[10] B. R. Wilcox and H. J. Lewandowski, Phys. Rev. PER 13, 020110 (2017).

[11] D. Hu, B. M. Zwickl, B. R. Wilcox, and H. J. Lewandowski, Phys. Rev. PER 13, 020134 (2017).

[12] B. M. Zwickl, T. Hirokawa, N. Finkelstein, and H. J. Lewandowski, Phys. Rev. PER 10, 010120 (2014).

[13] H. J. Lewandowksi, D. Bolton, and B. Pollard, PERC Proceedings, submitted (2018), arXiv:1807.03385.

[14] http://jila.colorado.edu/lewandowski.

[15] B. R. Wilcox and H. Lewandowski, Physical Review Physics Education Research 12, 010123 (2016).

[16] R. G. Lomax and D. L. Has-Vaughn, An Introduction to Statistical Concepts, 3rd ed. (Routledge, 2012). 